\section{NUEVA CENTRAL DEL OBERBANK SALZBURGO ALTA AUSTRIA}

$145-112$

\section{SINOPSIS}

En un privilegiado enclave urbano se ha construido esta central bancaria, de acuerdo con las más modernas técnicas organizativas y funcionales, y los últimos estudios sobre las formas de trabajo humanizadas en edificios administrativos.

En sus dos sótanos y siete plantas por encima de rasante, de distintas superficies - de acuerdo con sus diferentes usos y requisitos-, se han distribuido todas las dependencias necesarias en un edificio de este tipo, destacando en su organización la gran flexibilidad y funcionalidad de los diversos espacios conformados.

Mención aparte merece la especial atención prestada a la cimentación y estructura empleadas por la proximidad del Danubio; al tratamiento de las fachadas - dada la situación urbana de la construcción-, y a los sistemas de seguridad $e$ instalaciones por la función especifica del edificio.

El centro técnico del Oberbank puede considerarse no sólo como modelo de planificación urbana de la ciudad de Linz, sino que cumple, por su cometido y ejecución, todas las condiciones exigibles a una realización de este tipo.

El edificio se levanta sobre una parcela situada entre la plaza principal y una autopista y, además, entre dos calles que conducen a la zona urbana de Linz. Esta situación, favorable desde el punto de vista del tráfico, permite al cliente acceder cómodamente al edificio por vias muy transitables.

Ya en la fase de planificación se partió de los requisitos de un moderno edificio administrativo en relación, en sus aspectos organizativos y funcionales, con los principios del plan municipal y las necesidades de un ambiente de trabajo humanizado, teniendo en cuenta además que en el borde de la ciudad vieja, de carácter histórico, donde se encuentra el edificio, no se desean grandes masas de construcción. Estos requisitos se pudie-

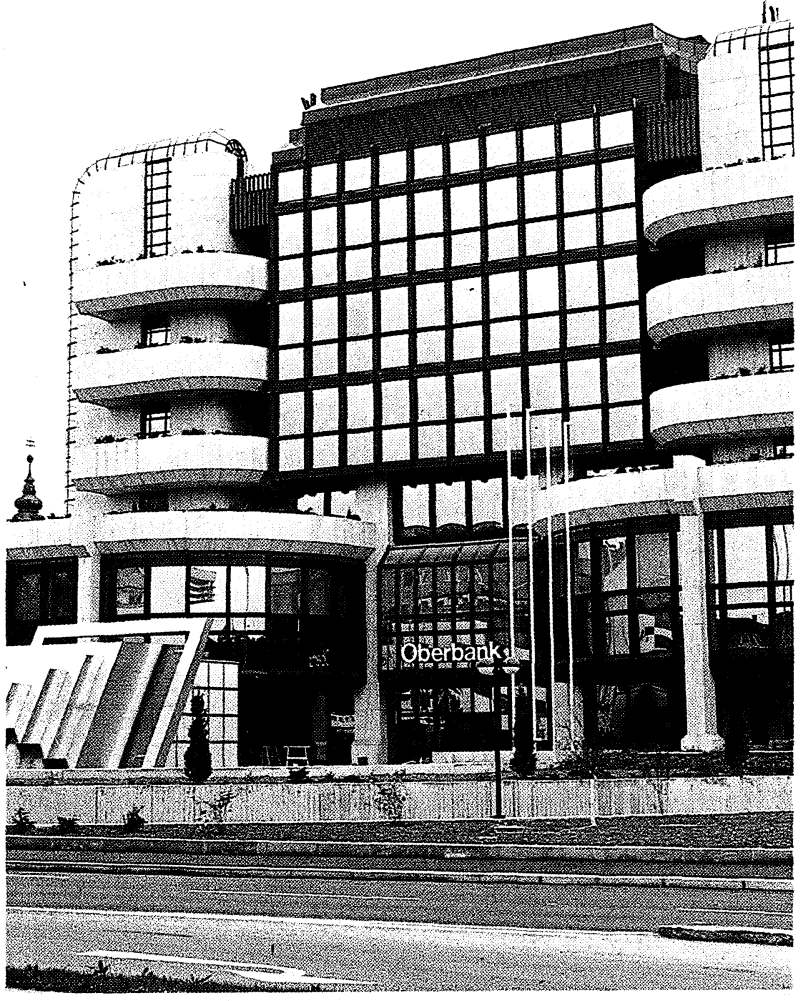

ron satisfacer de modo optimo desarrollando un cuerpo de construcción estilizado, de proporciones plásticas correctas.

En el diseño también influyó la posibilidad de ulteriores ampliaciones, ya tenidas en cuenta al elegir el terreno. Por consiguiente, al hacer la planificación y, posteriormente, la construcción, se previeron posibles ampliaciones a ambos lados del edificio, para satisfacer futuras necesidades.

El concepto espacial se basa en las exigencias técnicas y de organización del trabajo en oficina, en los conocimientos actuales acerca de la configuración de un lugar de trabajo humanizado, asi como en los requisitos de seguridad del Banco. Esto dió como resultado unas dependencias de gran flexibilidad en su configuración y utilización. Se emplearon abundantes elementos de compartimentación para evitar la impresión de grandes superficies, ya que se consideraba q'e la subdivisión del espacio subraya la intimidad y favorece el trabajo.

En la distribución de las distintas dependencias se buscó la especificidad de cada planta, tanto en relación con las demás como independientemente. Así, en el segundo sótano, se situaron 156 plazas de aparcamiento para el personal, almacenes, ar- 


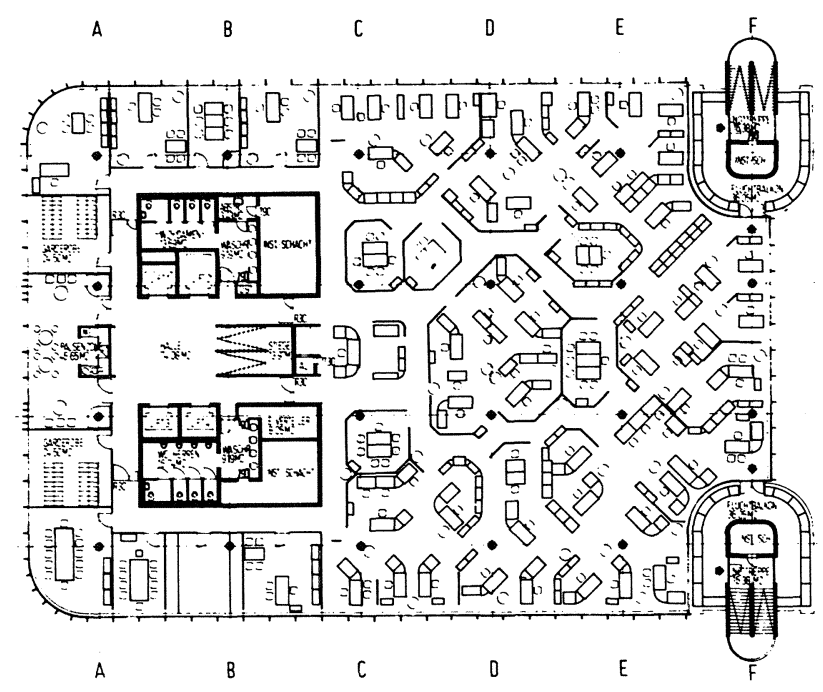

planta

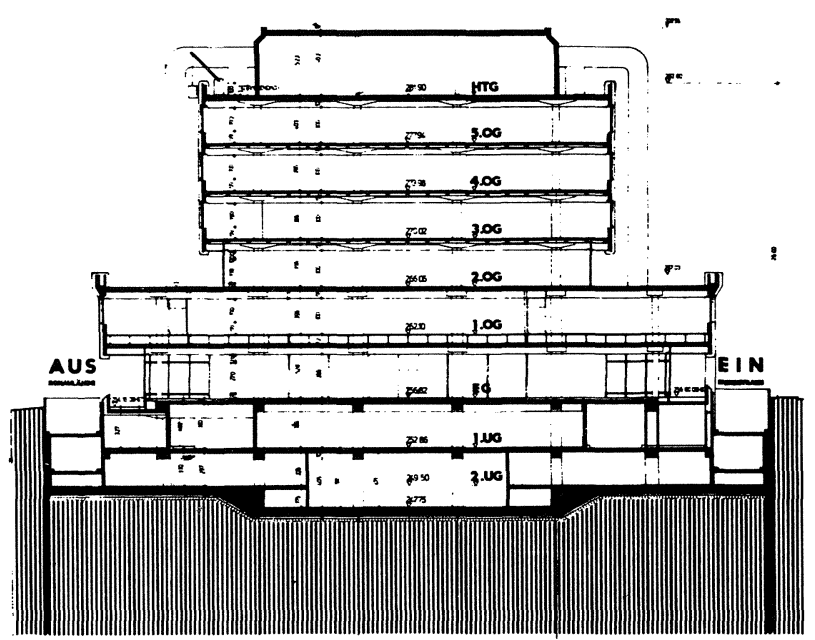

\section{sección}

chivos $y$, en la parte central rebajada, la central técnica de las plantas inferiores.

En el primer sótano se encuentran 72 aparcamientos para clientes, el grupo para el servicio de transporte, la imprenta, el almacén de material y la caja central con sus dependencias adicionales. Se puede acceder a ambos sótanos por medio de rampas que dejan separados los tráficos de entrada y salida.

En la planta baja se han dispuesto una sucursal del banco, parte del departamento extranjero, el departamento de administración y compra, asi como el de revisión interna. La entrada del público a la sucursal se efectúa por la plaza, mientras que el personal to hace por un pasaje privado.

En planta primera se distribuyen los departamentos de tráfico de pagos nacionales, el de correos y el de automoción.

La segunda planta sirve en su totalidad como planta social de la empresa. Cuenta con restauran- te, cocina con sus dependencias auxiliares, cafeteria, club, consulta médica, etc., contando la mayoria de ellas con una prolongación mediante terrazas al aire libre.

Las plantas tercera, cuarta y quinta se destinan exclusivamente a las oficinas privadas del banco.

Finalmente, por encima de la quinta planta y bajo el tejado, se han instalado los servicios técnicos de las plantas superiores.

La configuración regular de la planta muestra la concepción básica de todas las plantas de oficina. Situado excéntricamente en la zona suroeste se encuentra el núcleo de comunicación vertical, que consta de caja de escalera, grupo de cuatro ascensores, pozos de alimentación e instalaciones sanitarias. La poca anchura existente entre el núcleo y las fachadas se utiliza para dependencias individuales, salas de juntas, guardarropas y zonas de descanso. Las escaleras de incendio se encuentran en los extremos del edificio y constituirán el elemento de unión de las fachadas en el caso de ampliaciones posteriores.

La planta baja desempeña también la servidumbre de distribución para todos los demás grupos de funciones del centro técnico. El pasaje enlanza las dos rampas que conducen a los sótanos con el nivel de la calle, y abre el acceso, a través del montacargas, hasta los almacenes situados en el sótano.

En la segunda planta se encuentran únicamente las dependencias sociales para el personal. El restaurante de la empresa equivale, por su equipo y organización, a un moderno autoservicio con capacidad para 240 comensales. La cocina es una unidad muy eficaz que puede servir a un número todavia mayor de clientes. En la misma planta se encuentra también una cafetería, decorada al estilo vienés, con buffet anexo. Junto a la cafetería se sitúa un local para club que ofrece diversas alternativas a los trabajadores: libreria, zona de lectura, televisión y similares. La prolongación de estas zonas en terrazas ofrece un lugar de descan-

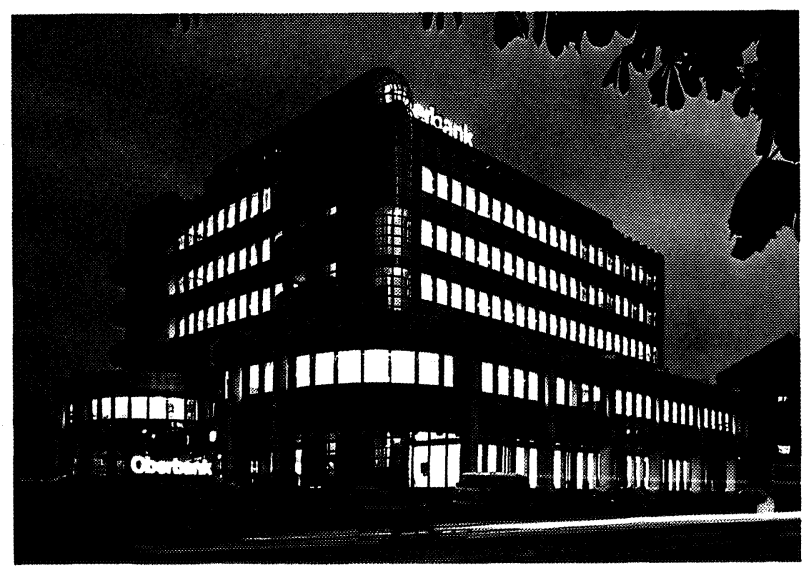


so adicional durante el verano. Conformadas como techo invertido con aislamiento térmico por la parte superior, presentan un suelo de placas de hormigón lavable, alternando con baldosas de grava y maceteros para plantas. Todos los antepechos están reverdecidos con jardineras. En la parte eștrecha de las terrazas, a ambos lados de la cafeteria, unas celosias de metal ligero sirven de protección contra el sol y de estructura portante para plantas trepadoras, mientras que, en la zona del restaurante, el voladizo de las plantas superiores de oficina proporciona suficiente defensa contra el soleamiento excesivo.

En la parte occidental de la primera planta se ha situado el grupo de dependencias para la formación del personal. Con el fin de poder utilizar este grupo para múltiples usos, se ha hecho gran hincapié en la flexibilidad de los mismos y, así, la mayor sala para actos puede subdividirse en tres más pequeñas utilizando tabiques móviles. Las instalaciones técnicas están dispuestas de modo que desde una cabina de proyección centralizada se puede proyectar a cada una de estas tres salas.

En el cuerpo oriental de la planta baja se encuentra una sucursal del banco, con acceso desde la parte norte de la casa. Los clientes de esta sucursal disponen de plazas de aparcamiento en el primer sótano del garaje, así como de aparcamientos para corto tiempo en las calles adyacentes. En la organización de esta sucursal se han seguido nuevas directrices: por ejemplo, no existen ventanillas ni cajas, como es lo usual, y en su lugar aparecen dos centros de servicio. El cometido de cada uno de éstos consiste en ofrecer al cliente todos los servicios necesarios desde un solo lugar, evitando, de esta forma, las diversas taquillas divididas por sectores. La entrega de dinero se realiza a través de un sistema de transporte neumático, en conexión con la caja central.

La concepción técnica de la nueva construcción se basa en una estructura realizada totalmente en hormigón armado, con pilares de hormigón hecho in situ y forjados distintos según las plantas: el del segundo sótano es de piezas prefabricadas

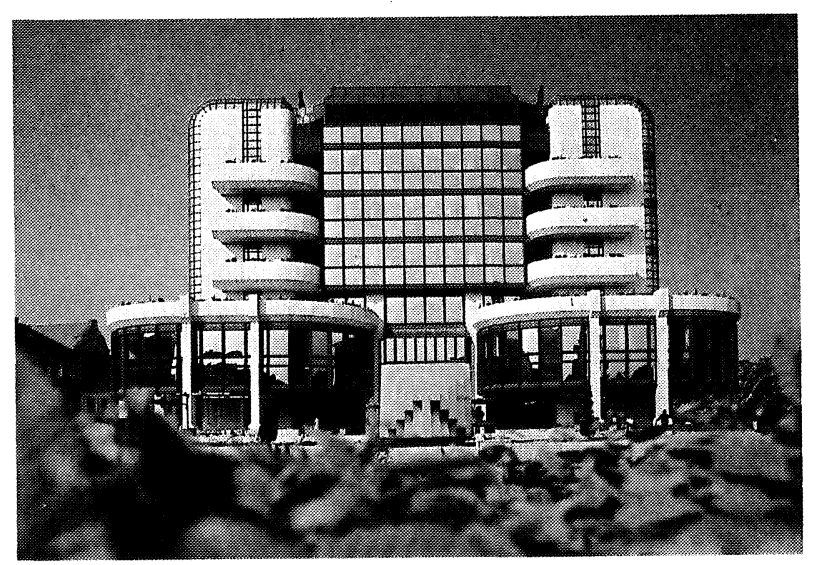

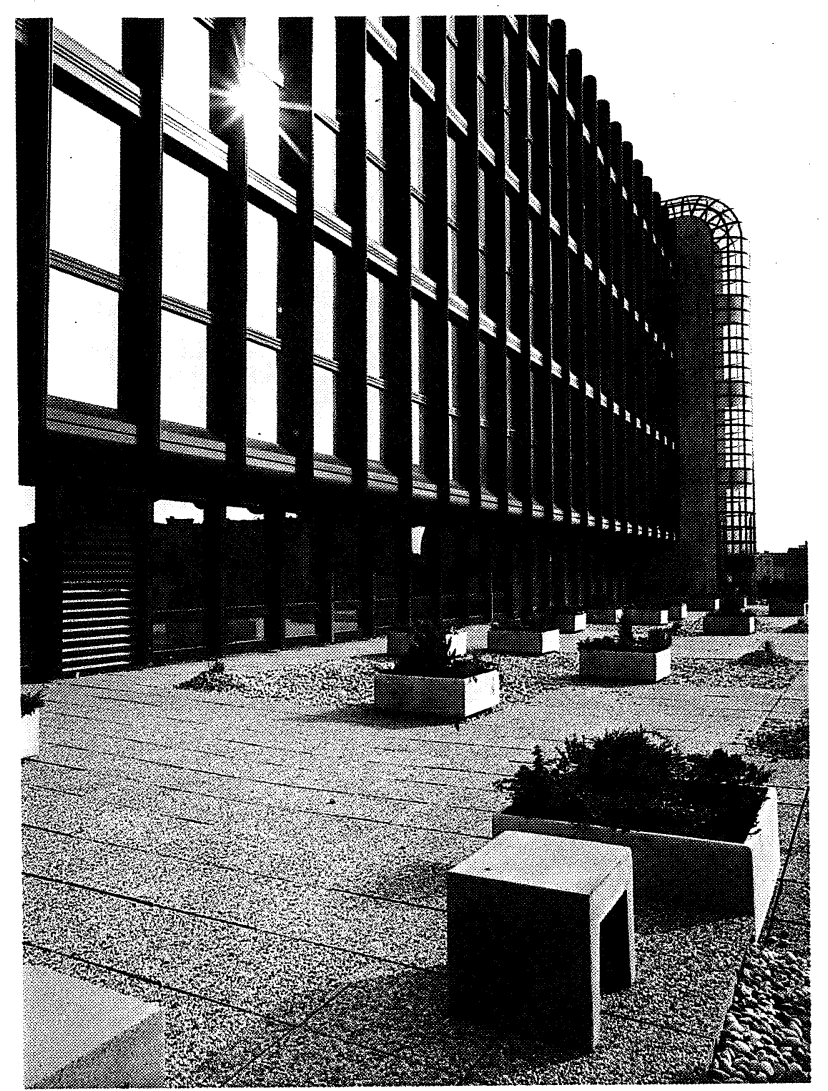

huecas; el del primer sótano es una losa de hormigón; en planta baja y primera se emplearon placas de hormigón pretensado; para los forjados de las plantas siguientes se eligieron, teniendo en cuenta las jácenas de apoyo en voladizo, también de hormigón, unos artesonados pretensados embutidos; por último, el techo por encima de la quinta planta está realizado con losas de hormigón hecho in situ. Todos los demás elementos portantes de la construcción también se hicieron de hormigón armado, como las cajas de escalera, los pozos de instalaciones verticales y los de ascensores, los muros de cerramiento de las zonas de seguridad, etc. En la configuración de la estructura se siguió un módulo reticular cuadrado de $8,25 \mathrm{~m}$ de lado.

La cimentación tuvo que realizarse extremando las precauciones por la proximidad inmediata de la zona ribereña del Danubio. Se emplearon grandes pilotes de hormigón, que llegan hasta las capas resistentes del terreno. Los dos sótanos presentan una superficie construida muy superior a la de la planta baja. El sentido de esta planificación no perseguía únicamente aumentar el número de plazas de aparcamiento en las plantas destinadas a garaje, sino también anticipar todos los trabajos de cimentación necesarios para una ulterior ampliación. La posible ampliación de los sótanos en fecha posterior, sólo sería posible enfrentándose a dificultades técnicas enormes y con un coste financiero también enorme, por la elevación del nivel freático que provocará el nuevo embalse de la 


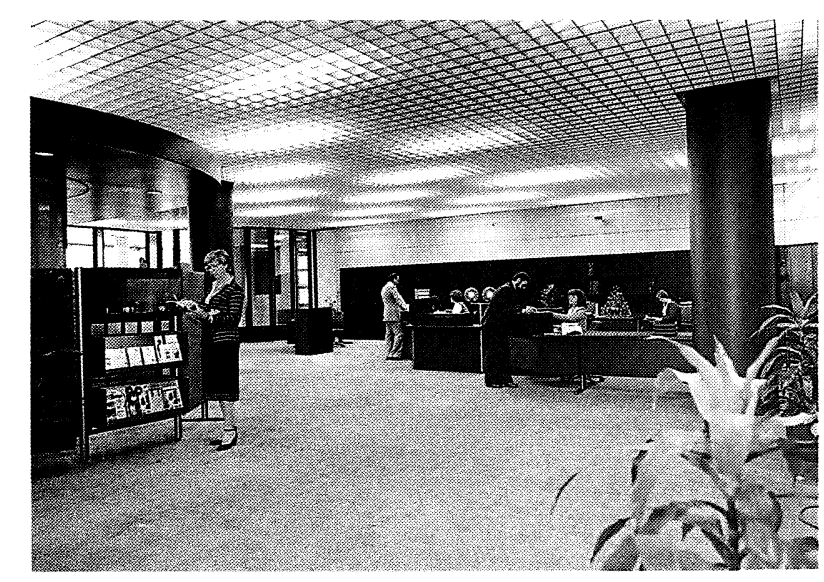

central eléctrica del Danubio. Como en estas zonas falta la sobrecarga de un edificio situado encima, se han utilizado pilotes de tracción para evitar la fuerza ascensional de los sótanos.

Teniendo en cuenta la situación urbana del edificio, en la elección de los revestimientos de las fachadas se tuvo en cuenta especialmente su aspecto exterior y su carencia de mantenimiento. Siguiendo estos principios se empleó un revestimiento de piedra de travertino en las columnas exteriores de las plantas baja y primera, en los antepechos de las terrazas y en la parte exterior de los núcleos de las escaleras de escape en la zona oriental. Las fachadas son de metal ligero, en color bronce oscuro, con ventilación por la parte posterior y colgadas de los forjados. Los antepechos son de hormigón armado, asimismo ventilados por atrás y con aislamiento térmico recubierto con vidrio esmaltado. Las ventanas disponen de cristal acústico y de persianas exteriores de maniobra eléctrica centralizada. El pavimento de alrededor del edificio en planta baja es de piedra artificial y, el que rodea la fuente, de baldosas de gravilla.

En el tratamiento interior de las plantas de oficina se han empleado: núcleos con piedra de travertino, tabiques de placas de cartón-yeso, falsos techos de metal ligero y suelos de moqueta. Todas las instalaciones se conducen por el suelo para dar servicio a cada puesto de trabajo. La planta

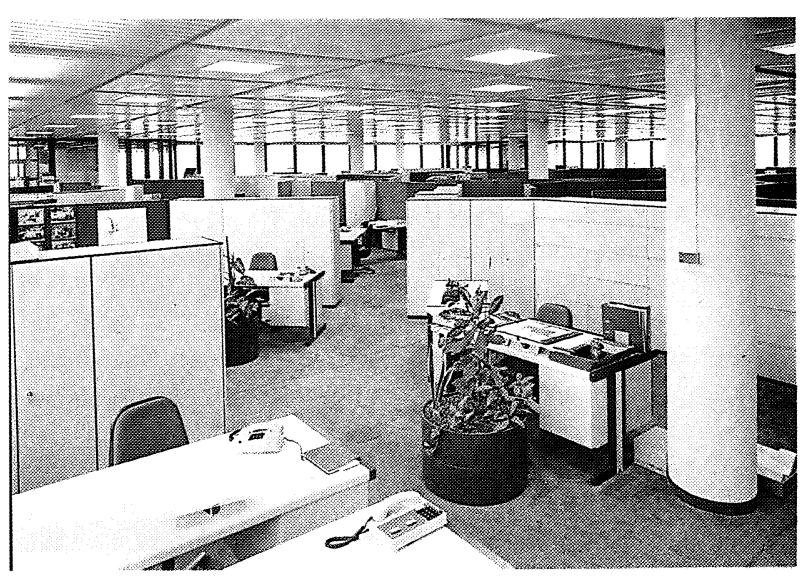

social, por su parte, emplea paredes de travertino, falsos techos reticulados y solados de madera.

Un factor esencial en la edificación lo constituyen los dispositivos de seguridad instalados, que no sirven solamente para proteger los valores depositados y los procesos de trabajo en el banco, sino también $y$, especialmente, al personal de la casa y a los clientes y visitantes. Según esto, se han dispuesto zonas de distintos grados de seguridad protegidas con instalaciones como el sistema de control de acceso, el sistema automático de detección de incendios, las vías de evacuación, etcétera.

Por último cabe mencionar las obras artísticas integradas en los distintos espacios del edificio. Así, por ejemplo, en el centro del vestíbulo se encuentra una fuente que sale de un estanque; es de acero y su dinámica la debe al agua que corre. Además es transitable y constituye el acceso a la zona de clientes del garaje del primer sótano. El hall de la entrada principal para el personal se ha adornado, en sus dos paredes longitudinales, con un friso metálico y un tapiz. Además, en el semicírculo de la escalera que va de la sala de cajas a la cámara acorazada de los clientes, un mural muestra la silueta del centro de la ciudad de Linz.

W. Schmidt 